\title{
Novel Acellular Scaffold Made from Decellularized Schwann Cell Sheets for Peripheral Nerve Regeneration
}

\author{
Radoslaw Junka $^{1} \cdot$ Xiaojun Yu ${ }^{1}$
}

Received: 29 June 2015 /Revised: 1 September 2015 / Accepted: 10 October 2015 /Published online: 4 November 2015

(C) The Regenerative Engineering Society 2015

\begin{abstract}
Extracellular matrix surrounding Schwann cells and neurons provides critical determinants of cellular phenotype during development as well as essential cues in stimulating and guiding regrowth. Using cell sheet technology, we developed a novel scaffold enriched with native extracellular matrix from Schwann cells. Schwann cells were grown into sheets and layered onto polycaprolactone fibers for support. Upon decellularization of these constructs, extracellular matrix remained with few traces of nucleic acids. This method of deposition of extracellular matrix provided more protein than traditional seeding method after decellularization. Additionally, the isolated matrix supported proliferation of Schwann cells better than covalently bound laminin. The proliferation and differentiation of Schwann cells grown on decellularized sheets were complemented by upregulation of Erbb2 and myelin protein zero. Laminin expression of $\beta 1$ and $\gamma 1$ chains was also elevated. PC12 cells grown on decellularized sheets produced longer neurite extensions than aligned polycaprolactone fibers alone, proving potential of these scaffolds to be used in future peripheral nerve regenerative studies.
\end{abstract}

Electronic supplementary material The online version of this article (doi:10.1007/s40883-015-0003-2) contains supplementary material, which is available to authorized users.

Xiaojun $\mathrm{Yu}$

xyu@stevens.edu

1 Department of Chemistry, Chemical Biology and Biomedical Engineering, Stevens Institute of Technology, Hoboken, NJ 07030, USA

\section{Lay Summary}

Peripheral nerve injuries present a serious clinical need with approximately $50 \%$ of surgical cases achieving only some restoration of function. In order to better guide regenerating nerves, supporting cells of the nerve tissue were grown into sheets and subsequently decellularized, leaving a myriad of surrounding protein as a scaffold. Constructs have been shown to support cell growth and neurite extension in vitro. Future projects will combine various cell types present in the nerve tissue as well as stem cells to fully support and reconstruct architecture of the peripheral nerves.

Keywords Extracellular matrix $\cdot$ Schwann cell $\cdot$ Nerve tissue engineering $\cdot$ Cell sheet $\cdot$ Decellularization

\section{Introduction}

Schwann cells are the principal neuroglia of the peripheral nervous system. They promote cell attachment, proliferation, axon ensheathment, axon myelination, and growth factor secretion. During nerve development and regeneration process, Schwann cells deposit extracellular matrix (ECM), comprised from a diverse set of macromolecules, such as collagens, laminins, and proteoglycans [1]. The ECM provides physical support for the cellular component of the tissue and initiates biochemical and biomechanical signals that required for tissue morphogenesis, differentiation, and homeostasis [2]. For example, laminins provide structure to the basement membrane of a nerve, serve as an attachment site for cells via cell surface protein, and act as ligands for integrins on cell surface to initiate signal transduction pathways [3, 4]. Recently, studies have used xenogeneic and allogeneic ECM as a source for a natural scaffold in both preclinical and human clinical studies for the reconstruction of tissues [5]. According to "dynamic 
reciprocity" theory, ECM is capable of exerting an influence on gene expression via transmembrane proteins and cytoskeletal components. This in turn is thought to affect rates of transcription, mRNA stability, and rates of translation and posttranslational modifications [6]. Thus, by enhancing deposition and composition of the ECM, Schwann cell regenerative processes may be indirectly enhanced and subsequently result in faster nerve regeneration.

After peripheral nerve injury, Schwann cells promote nerve regeneration by producing the regeneration-promoting matrix as well as growth factors. More importantly, if axonal contact is lost, the Schwann cells dedifferentiate and proliferate, myelin-related genes are downregulated, while adhesion molecules, neurotrophins, cytokines, and their receptors are upregulated to create an environment that allows axonal regrowth [7]. Transplantation of cultured Schwann cells into peripheral nerve grafts has been shown to improve nerve regeneration in experimental models of peripheral nerve injury [8-10]. However, its clinical application remains controversial due to the difficulty of isolation, expansion, and purification for Schwann cells in vitro, as well as the immunoreaction [11]. In order to bypass the immunosuppression and donor site morbidity in autologous transplants, various methods of decellurizing allogenic nerves have been performed [12]. The chemical extraction of the acellular allogenic nerves allows for preservation of the basal lamina tubes and the threedimensional collagen scaffolding present within native nerve [13]. This three-dimensional structure that provides guidance cues to regenerating axons is absent within artificial conduits [14-16]. Decellularization makes possible to remove myelin and cell debris, rendering the acellular allograft less immunogenic than cellular allografts $[17,18]$. Nonetheless, the inherent limitation of these transplantation techniques is the limited number of available donor grafts [12]. Although, artificial nerve conduit transplants are not limited by the availability of transplantable tissue, the main limitation with these artificial conduits is a time constraint to produce a "custom" conduit and the inability to fully recapitulate complex extracellular environment present in a nerve. These structures are not very good at creating multi-phenotypic cell-dense tissues such as nerve. They often cause fibrosis creating an impulse conduction barrier and misguidance of the axons $[19,20]$. Thus, they are inefficient in bridging peripheral nerve lesions with long or extended gaps [21, 22].

Use of thermo-responsive substrates that generate sheets of cells embedded in a native ECM may provide an alternative to generating artificial conduits containing many of the extracellular cues for axonal regeneration. The cell sheet technology makes use of controllable hydrophobicity of a polymer, poly( $N$-isopropylacrylamide), that is deposited on the surface of a petri dish, which enables reversible cell adhesion to and detachment from the dish [23]. Cultured cells can be noninvasively harvested as an intact monolayer cell sheet along with its surrounding ECM. Layering of these monolayers provides an opportunity to construct clinically relevant in size constructs as well as introduce multiple cell types present in the native nervous tissue [24]. This technology has been used to reproduce many tissues including the skin [24], corneal epithelia [25, 26], and kidney glomeruli [27, 28]. Schwann cells have been shown to form monolayers on these substrates [29]. By taking advantage of cell sheet technology, a conduit composed of Schwann cells' axon-guiding ECM can be assembled from allogenic source. In combination with the decellularization process, this method can bypass timeconsuming isolation, expansion, and purification from an autologous source, rendering a non-immunogenic allogenic scaffold containing guidance cues present in the native nerve. While current fabrication techniques allow for only few molecular cues to be incorporated into the scaffold, this method uses cells as "natural factories" for deposition of various cellsecreted peptides as well as their transcript variants. Our novel approach addresses this important limitation and focuses genetic expression profile of Schwann cells grown on these regenerative matrices.

\section{Materials and Methods}

All chemicals used for nanofibrous scaffold preparation and cell sheet decellularization were purchased from SigmaAldrich unless otherwise indicated. 1,1,1,3,3,3-Hexafluoro2-propanol (HFIP) was purchased through Oakwood Products. All cell culture reagents including Dulbecco's modified Eagle's medium (DMEM), fetal bovine serum (FBS), penicillin streptomycin, and trypsin were purchased from Life Technologies. Nunc UpCell $60-\mathrm{mm}$ dishes were purchased from Thermo Scientific. Analytical reagents were purchased from following sources: CellTiter $96^{\circledR}$ AQueous Non-Radioactive Cell Proliferation Assay (MTS) (Promega), Coomassie Blue R250 (Bio-Rad), Quant-iT ${ }^{\mathrm{TM}}$ PicoGreen ${ }^{\circledR}$ dsDNA (Invitrogen), BCA assay kit (Thermo Scientific), RNeasy Plus Mini Kit (Qiagen). All reagents used in PCR analysis were purchased through Promega Co. Laminin and the primary monoclonal antibody for neurofilament-M+H mouse monoclonal antibody were purchased through Invitrogen and secondary FITC-conjugated anti-mouse antibody was purchased through Jackson Immunoresearch.

PCL fibers were solely used as a mechanical support of ECM throughout the decellularization process. Layering cell sheets on glass alone tended to rip cell sheets apart. Moreover, the decellularization protocol disintegrated cell sheets without any mechanical support. Electrospun poly-caprolactone (PCL) fibers were fabricated using a blend PCL dissolved in HFIP at a total concentration of $16 \%(w / v)$ and stirred overnight. PCL/chitosan scaffolds were electrospun from a solution blend of $1 \%$ chitosan dissolved in acetic acid and $32 \%$ 
PCL solution in HFIP magnetically stirred for $2 \mathrm{~h}$. Briefly, solutions were electrospun using a high voltage of $12 \mathrm{kV}$ between the solution and the receiving apparatus with a constant distance of $8 \mathrm{~cm}$. Solutions were delivered with a $5-\mathrm{ml}$ polypropylene syringe through a $0.9-\mathrm{mm}$ tip needle at a flowing rate of $0.25 \mathrm{ml} / \mathrm{h}$ using a syringe pump. Fibers were collected onto a flat plate coating a layer of aluminum foil to form meshes and collected onto a die steel mandrel of $18 \mathrm{~cm}$ in diameter with a rotating rate of $1000 \mathrm{rpm}$ to form aligned fibers. All electrospun fibers were sterilized with isopropanol as well as ultraviolet lamp for $2 \mathrm{~h}$ and washed three times with phosphate-buffered saline (PBS) prior to cell culture.

Rat Schwann cells were cultured in high-glucose DMEM containing $10 \%$ FBS, 100 units $/ \mathrm{ml}$ penicillin, and $100 \mu \mathrm{g} / \mathrm{ml}$ streptomycin. Cells were seeded at the density of $5 \times 10^{5}$ and were grown into sheets for 7 days on Nunc UpCell $60-\mathrm{mm}$ dishes with media change every other day. At the conclusion of culture, UpCell petri dishes were removed out of the incubator to room temperature in order to induce lifting of a cell sheet. Sheets were maneuvered onto a scaffold and covered it uniformly. Cells were allowed to attach to the scaffold for $2 \mathrm{~h}$ with minimal media and cultured overnight after addition of more media. For a detailed description, please see Online Resource 2. For stacking cell sheets, each layer was added after a 2-h period.

The ECM was obtained using a modified protocol for nerve decellularization [30]. Briefly, a combination of sodium deoxycholate, SB-10, and SB-16 was selected as detergents for decellularization experiments. Scaffolds with attached cell sheets were washed with $10 \mathrm{mM}$ phosphate-buffered $50 \mathrm{mM}$ sodium solution. All washing steps were carried out at $4{ }^{\circ} \mathrm{C}$ with agitation to prevent protein cleavage. After $2 \mathrm{~h}$, the solution was aspirated, replaced with a $10 \mathrm{mM}$ phosphate-buffered $50 \mathrm{mM}$ sodium solution containing $125 \mathrm{mM} \mathrm{SB}-10$, and agitated overnight. The tissue was then rinsed once for $5 \mathrm{~min}$ in a $50 \mathrm{mM}$ phosphate-buffered $100 \mathrm{mM}$ sodium solution. The washing buffer was replaced with a $10 \mathrm{mM}$ phosphatebuffered $50 \mathrm{mM}$ sodium solution containing $0.6 \mathrm{mM}$ SB-16 and $0.14 \%$ sodium deoxycholate. After agitating overnight, the tissue was rinsed with the washing buffer three times at 5 min per rinse. The scaffolds underwent this process two times to ensure maximum cell debris removal. The scaffolds were stored in a $10 \mathrm{mM}$ phosphate-buffered $50 \mathrm{mM}$ sodium solution containing protease inhibitor cocktail at $4{ }^{\circ} \mathrm{C}$ [30].

Crosslinking experiments were designed to immobilize laminin via its carboxy group to amine groups in chitosan via EDC/NHS mechanism [31]. Solutions of laminin 1:100 $(v / v)(1 \mathrm{mg} / \mathrm{ml})$ and $0.1 \mathrm{M}$ MES were prepared and mixed with ethylene diaminecarbodiimide (EDC) $(8 \mathrm{mg} / \mathrm{ml})$ and $n$ hydroxysuccinimide (NHS) $(22 \mathrm{mg} / \mathrm{ml})$. The resulting solution was agitated for $1 \mathrm{~h}$ under room conditions. The solution was then transferred onto fibers and allowed to crosslink overnight, at $4{ }^{\circ} \mathrm{C}$. The scaffolds were then washed with sterile
PBS thrice to obtain functionalized scaffolds. As a control, scaffolds without any modifications (plain PCL-chitosan and plain PCL) were also prepared.

Methylene blue (MB) and Coomassie Blue R250 stains were used to examine DNA and protein content, respectively. Decellularized scaffolds were agitated in $0.05 \% \mathrm{MB}$ solution for $20 \mathrm{~min}$ and washed extensively in PBS until no more dye diffused. Scaffolds washed in Coomassie Blue stain were treated for $1 \mathrm{~h}$ and washed in PBS extensively until no more dye diffused. Scaffolds with no matrix were used as controls. Images were captured using Nikon T100 stereomicroscope. Prior to BCA and PicoGreen assays, scaffolds were detached from cover slips. After the addition of $5 \mathrm{ml}$ of working solution, scaffolds were vortexed for $1 \mathrm{~min}$. Each assay was performed according to manufacturer's protocol. Scaffolds with a single and three cell sheet layers were evaluated. Each scaffold was represented in triplicate. Standard curve for each assay was established during each reading.

The proliferation MTS assay was done after 4 days of culture. After decellularization, scaffolds were sterilized with $70 \%$ ethanol for $1 \mathrm{~h}$ under laminar flow hood. Ultraviolet light sterilization was avoided. Scaffolds were rinsed three times in PBS and placed in a complete DMEM medium. Schwann cells were seeded onto cell sheets attached to random PCL meshes at a density of $2.1 \times 10^{5}$ cells/well and cultured in full supplemented DMEM. Cells seeded on PCL nanofibers were used as controls. As additional controls, PCL/chitosan fibers and PCL/chitosan fibers with crosslinked laminin were used. To test influence of the fiber alignment, random and aligned scaffolds were used for each condition. The assay was performed according to manufacturer's protocol. Absorbance was measured at $490 \mathrm{~nm}$ using a using a microplate reader (Synergy ${ }^{\mathrm{TM}}$ HT, BioTek Instruments, Inc.). Each group contained ten samples and the test was conducted three times.

For RT-PCR experiments, 500 ng of total RNA was extracted from Schwann cell cultures using RNeasy Plus Mini Kit. The concentration and quality RNA was evaluated by measuring absorbance ratio 260/280. Only RNA with absorbance ratio is of 2.0 was used for the experiments. The cDNA synthesis was carried out using M-MLV Reverse Transcriptase, according to the manufacturers' instructions. For DNA amplification, 30 cycles were used as follows: $95^{\circ} \mathrm{C}$ for $15 \mathrm{~s}$ for denaturation, $65^{\circ} \mathrm{C}$ for $1 \mathrm{~min}$ for primer annealing, and $72{ }^{\circ} \mathrm{C}$ for $1 \mathrm{~min}$ for extension. Primer sequences and accession numbers are listed in Online Resource 1. The primer specificity was analyzed using gel electrophoresis. Each agarose gel was prepared at $2 \%(w / v)$ in order to resolve 200-bp fragments. Images of the ethidium bromide-stained agarose gels were acquired with Canon PowerShot S110 12.1 MP digital camera. Quantification of the bands was performed by ImageJ software. Band intensity was expressed as relative absorbance units. Mean and standard deviation of all experiments 
performed were calculated after normalization to beta actin $[32,33]$.

In immunofluorescence, non-adherent rat PC-12 (pheochromocytoma cell line) was stained using neurofilament$\mathrm{M}+\mathrm{H}$ mouse monoclonal antibody. $\mathrm{PC} 12$ cells were seeded onto scaffolds at about $1 \times 10^{2}$ cell clumps/well and were supplemented each day with NGF (Sigma) at a final concentration of $100 \mathrm{ng} / \mathrm{ml}$ for 10 days. Cells were then fixed for $30 \mathrm{~min}$ using $4 \%$ paraformaldehyde and permeabilized with $5 \%$ goat serum containing PBS with $0.5 \%$ Triton ${ }^{\circledR} \mathrm{X}-100$ for $30 \mathrm{~min}$. Fixed/permeabilized samples were blocked using $5 \%$ goat serum dissolved in PBS for $1 \mathrm{~h}$. Samples were then incubated with primary antibody overnight. FITC-conjugated antimouse IgG was used as secondary antibody for $30 \mathrm{~min}$. Samples were mounted with Fluoroshield ${ }^{\mathrm{TM}}$ with DAPI (Sigma) and visualized using a Zeiss LSM5 Pascal confocal microscope (Zeiss). Five randomly selected fields for each condition were imaged for neurofilament measurement. PC12 cells grown on randomly aligned fibers with decellularized sheet and aligned PCL fibers alone were used as controls.

All quantitative data are reported as mean \pm standard deviation. At least three samples per time point per scaffold type were evaluated for statistical analysis. Statistical differences among the groups of scaffolds were determined by performing a one-way ANOVA with post hoc Tukey's test using GraphPad Prism software. A confidence interval of $p<0.05$ was considered to be statistically significant.

\section{Results}

To examine decellularization process and its downstream applicability, it is necessary to evaluate the amount of matrix that can be isolated from a single as well as multiple sheets and the DNA content of these scaffolds. Presence of nucleic material has been directly correlated to adverse host reactions [34]. Purple Coomassie Blue staining of decellularized sheets indicated presence of protein (Fig. 1a-b). A visible nanofibrous scaffold in Fig. 1a proves that the staining was due to presence of the protein within the decellularized the sheet and not fibers. Faint blue MB staining (Fig. 1c, d) indicates low levels of DNA in the scaffold. Lack of defined dark blue structures indicates absence of nuclei. To determine applicability of cell sheets as an ECM source for the neuronal scaffolds, staining was used to compare cell sheets and traditional seeding method. Decellularized scaffolds (Fig. 1a) had more intense purple staining than traditionally seeded scaffolds (Fig. 1e). In Fig. 1b, neither individual cells nor PCL fibers were visible under higher magnification. Traditionally seeded scaffolds that were decellularized (Fig. 1f) show individual cells stained in purple as well as PCL fibers underneath. In Fig. 1c, d, faint blue staining of decellularized sheets indicates presence of the DNA within the construct. Traditionally seeded scaffolds

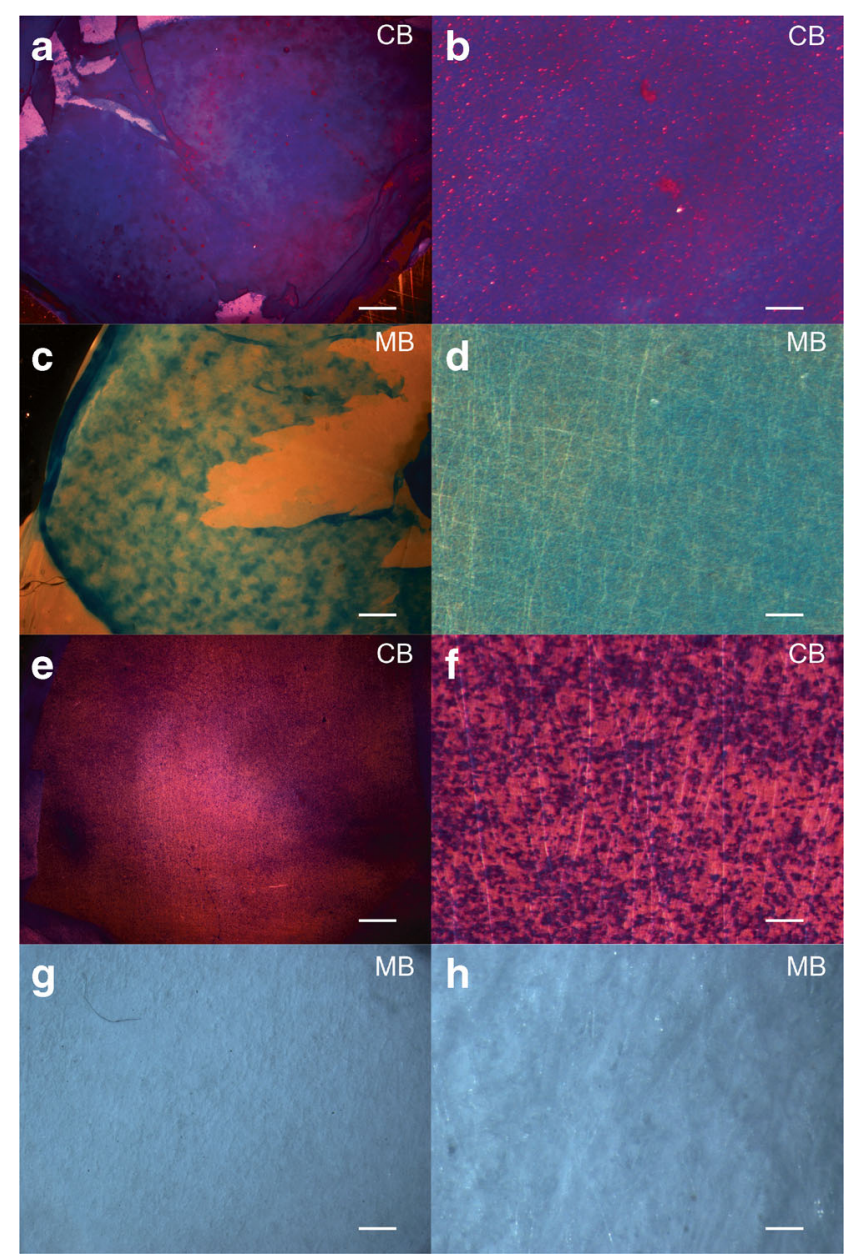

Fig. 1 a-b Intense purple staining of protein on decellularized Schwann cell sheets using Coomassie Blue stain. $\mathbf{c}-\mathbf{d}$ Faint blue staining of nucleic acids showing lack of nuclei structures remaining on decellularized Schwann cell sheets using Methylene blue stain. e-f Intense purple staining using Coomassie Blue for protein remaining after decellularization of traditionally seeded Schwann cells. $\mathbf{g}-\mathbf{h}$ Very diffuse Methylene blue staining showing small traces of nucleic acids on decellularized scaffolds traditionally seeded with Schwann cells. Scale bars represent $1 \mathrm{~mm}(\mathbf{a}, \mathbf{c}, \mathbf{e}, \mathbf{g})$ and $100 \mu \mathrm{m}(\mathbf{b}, \mathbf{d}, \mathbf{f}, \mathbf{h}) . M B$ Methylene blue, $C B$ Coomassie Blue

(Fig. 1g, h) show even weaker blue staining and absence of any defined nuclear structures.

Results obtained with Coomassie Blue staining were quantified using BCA assay (Fig. 2a). Scaffolds seeded traditionally were used as a control. To illustrate advantage of the cell sheet constructs, three cell sheets were layered and decellularized. Scaffolds composed of three cell sheets had an average protein content of $6.0402 \pm 1.11 \mathrm{mg}$. Constructs composed of a single cell sheet contained about three times less protein $1.9212 \pm 0.23 \mathrm{mg}$. Scaffolds that were seeded traditionally had a protein content of $1.5461 \pm 0.32 \mathrm{mg}$. Thus, stacking cell sheets significantly increased protein content of the nanofibrous scaffold. No statistical significance was observed between traditionally seeded constructs and single decellularized cell sheet. To confirm results obtained using 

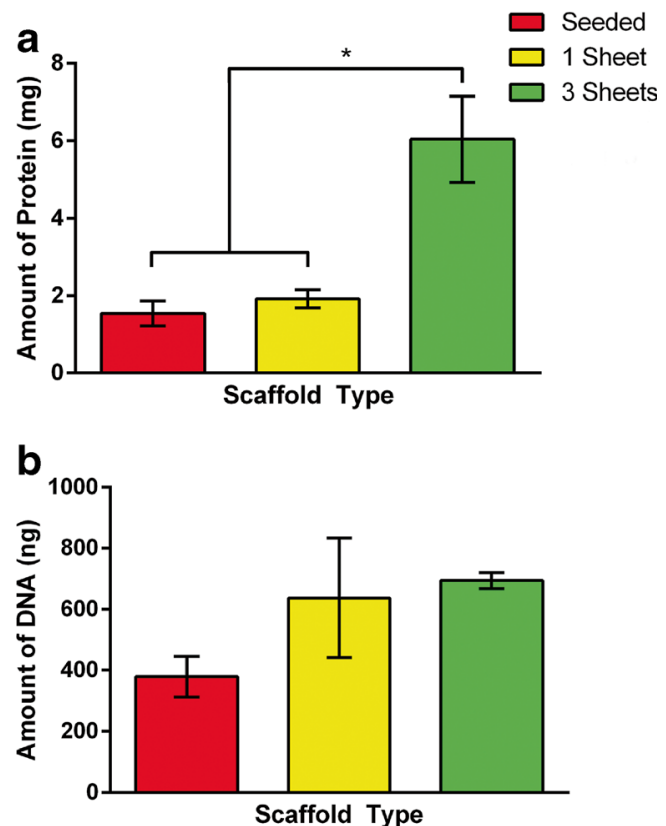

Fig. 2 a Quantification of protein remaining on a scaffold after decellularization protocol using BCA assay. Scaffolds were either seeded using traditional method, layered with a single decellularized sheet, or layered with three decellularized sheets $(* p=0.0003)$. b Quantification of nucleic acids remaining on a scaffold after decellularization protocol using QuantiT assay

MB staining, QuantiT assay (Fig. 2b) was used to quantify the amount of nucleic acids on each construct. Traditionally seeded scaffolds had a nucleic acid content of $378.03 \pm 94.33 \mathrm{ng}$. Scaffolds with a single decellularized cell sheet had nucleic acid content of $636.97 \pm 195.86 \mathrm{ng}$. Constructs with three decellularized cell sheets had nucleic acid content of 693.80 \pm 25.54 ng. Stacking decellularized sheets increased nucleic acid content in comparison to traditionally seeded constructs. No statistical significance was observed between traditionally seeded constructs and single decellularized cell sheet.

In MTS experiments (Fig. 3) cultures grown on random and aligned PCL scaffolds had absorbance of $0.733 \pm 0.097$ and $0.621 \pm 0.092$ O.D., respectively. Cultures grown on random and aligned PCL/chitosan scaffolds had absorbance of

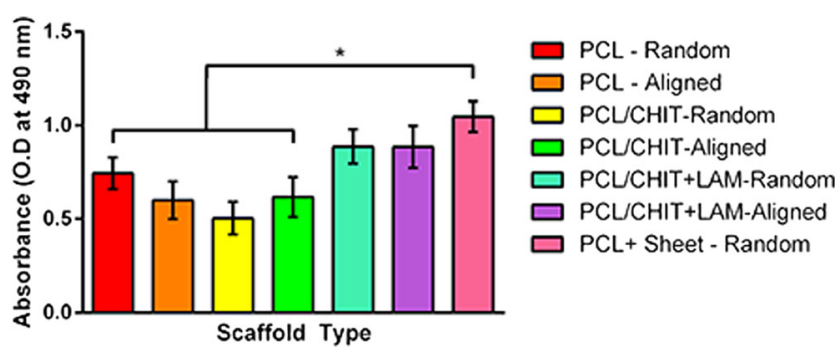

Fig. 3 MTS proliferation assay of Schwann cells seeded on various surfaced. $P C L / C H I T+L A M$ fibers indicate covalent modification of fibers using EDC/NHS chemistry. $P C L+$ SHEET indicate a single decellularized sheet attached to random polycaprolactone fibers. $\left({ }^{*} p<0.0001\right)$
$0.554 \pm 0.11$ and $0.612 \pm 0.099$ O.D., respectively. These absorbance values were significantly less than absorbance values of $1.056 \pm 0.096$ O.D. for constructs with a single decellularized Schwann cell sheet. Random and aligned PCL/chitosan nanofibrous scaffolds with covalently bound laminin had absorbance values of $0.900 \pm 0.098$ and $0.898 \pm 0.115$ O.D., respectively. Although average values of constructs with laminin attached were less than values of decellularized cell sheet, no statistical difference was shown between these constructs.

Using RT-PCR, several characteristic genes found to be expressed in Schwann cells were screened. Expression levels for collagen type IA and type $2 \mathrm{~A}$, fibronectin, pleiotrophin, NGF, BDNF, NT-3, neuregulin 1 and 2, and dystroglycan did not show any statistical difference (data not shown). However, Schwann cells grown on decellularized cell sheets showed a significantly elevated expression levels for Erbb2 (Fig. 4b) $0.643 \pm 0.021$ a.u., in comparison to cells grown on random and aligned PCL fibers, $0.450 \pm 0.023$ and $0.522 \pm 0.006$ a.u., respectively. Schwann cells grown on decellularized cell sheets also showed significantly elevated levels of expression for Laminin $\beta 1$ and $\gamma 1$ chains (Fig. 4c, e) with normalized intensity levels of $0.965 \pm 0.025$ and $0.929 \pm 0.032$ a.u., respectively. Expression levels for laminin $\beta 1$ and $\gamma 1$ chains in cultures grown on random and aligned PCL fibers were $0.828 \pm 0.029$ and $0.826 \pm 0.015$ a.u. as well as $0.866 \pm 0.002$ and $0.836 \pm 0.011$ a.u., respectively. Laminin $\alpha 2$ chain expression (Fig. 4a) did not show significant upregulation between decellularized scaffolds, $0.776 \pm 0.036$ a.u., or random and aligned nanofibrous scaffolds, $0.851 \pm 0.046$ and $0.824 \pm$ 0.086 a.u., respectively. Expression of $\beta 1$ intergin (Fig. 4f) was also shown to be elevated in cultures grown on decellularized sheets, $0.660 \pm 0.0627$ a.u. Cells grown on random and aligned fibers without the decellularized sheet have shown expressions levels of $0.449 \pm 0.0128$ and $0.367 \pm$ 0.0545 a.u., respectively. Schwann cells grown on decellularized cell sheets showed significantly elevated levels of expression, $1.272 \pm 0.063$ a.u., for myelin protein zero (P0) (Fig. 4d). Cells grown on random and aligned PCL nanofibrous scaffolds showed expression of $1.020 \pm 0.048$ and $1.073 \pm 0.024$ a.u., respectively.

PC12 cells grown on aligned fibers with decellularized sheets (Fig. 5a) had fewer neurite extensions than cells grown on random fibers with decellularized sheets or aligned fibers with no cell sheet (Fig. 5b, c). Neurite extensions also tend to lose directionality on aligned fibers with decellularized sheets in comparison to cultures grown on aligned fibers alone (Fig. $5 \mathrm{c}$ ). Neurite extension was quantified in Fig. 5d. Neurite extensions were significantly longer on constructs with aligned fibers and decellularized sheets with an average length of $696.2 \pm 30.1 \mu \mathrm{m}$. In comparison, neurite extensions on random fibers with decellularized sheets and aligned fibers alone had length of $317.7 \pm 37.4$ and $569.3 \pm 11.3 \mu \mathrm{m}$. 
Fig. 4 Genetic expression of Schwann cells seeded on decellularized Schwann cell sheets: a laminin chain $\alpha 2$ $\left({ }^{*} p=0.5322\right)$, b receptor tyrosineprotein kinase erbB-2 $\left({ }^{*} p<0.0001\right), \mathbf{c}$ laminin chain $\beta 1$ $\left({ }^{*} p=0.0007\right), \mathbf{d}$ myelin protein zero $(* p=0.0015)$, e laminin chain $\gamma 1(* p=0.0020)$, and $\mathbf{f} \beta 1$ integrin $\left({ }^{*} p=0.0193\right)$

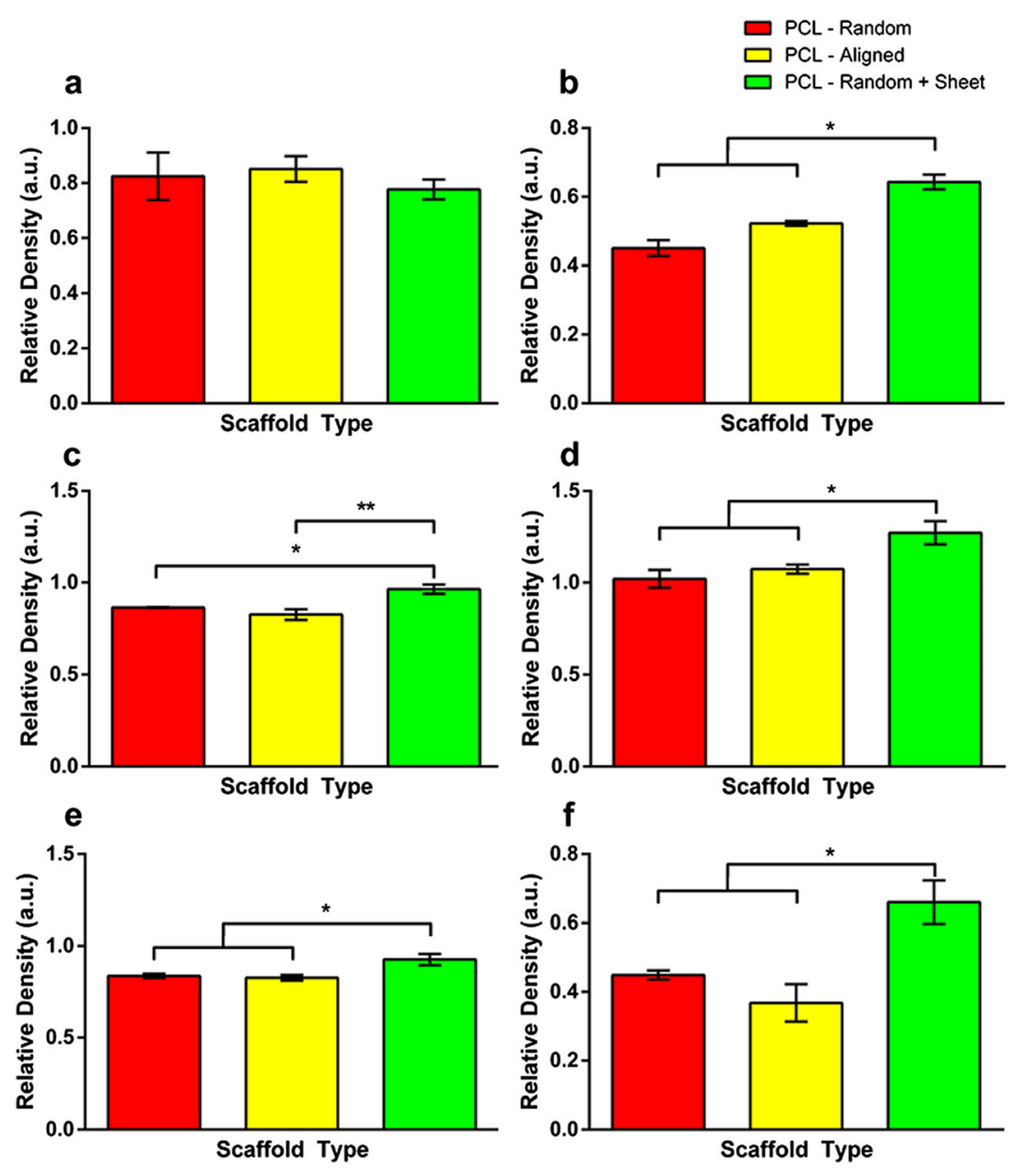

\section{Discussion}

ECM plays a dual role in tissue development and regeneration. It provides essential physical scaffolding for cells and initiates crucial biochemical and biomechanical processes required for tissue morphogenesis, differentiation, and homeostasis. However, it is difficult to construct a regenerative scaffold that would include a myriad of proteins, carbohydrates, and proteoglycans present in the native tissue. As a result, decellularization of organs and tissues has been used as a way to produce scaffolds composed of biological material. Nonetheless, availability of grafts to be decellularized is a key drawback. We here propose a novel method to produce an allogenic ECM scaffold for peripheral nerve regeneration. By using cell sheet technology, we were able to grow Schwann cell into stackable sheets, with nanofibrous scaffolds for structural support. Upon attachment of cell sheets to the scaffold, the sheets were treated with mild detergents to remove cells. To evaluate the effectiveness of the decellularization protocol, scaffolds were stained with Coomassie Blue for protein as well as with MB for nucleic acids. Coomassie Blue staining in Fig. 1a, b showed intense purple staining indicating presence of an intact ECM after the detergent treatment. Coomassie Blue staining does not reliably predict protein content in the scaffold since presence of carbohydrates interferes with binding of the compound [35]. A weak MB staining in Fig. 1c, d indicated that some nucleic acids remained on the scaffold. Scaffolds with content of $250 \mathrm{ng}$ and length of $250 \mathrm{bp}$ have been shown to cause immune response upon implantation [5]. It is unknown whether a scaffold with this level staining would cause an immune response. The detergent treatment could be improved by addition of DNAse and RNAse. Weak staining could also be accounted by the presence of sulfated carbohydrate moieties [36].

Using cell sheet technology for scaffold construction allows for subsequent stacking cell sheets and fabrication of constructs more relevant in size and mass. Despite same seeding density of $5 \times 10^{5}$ cells, decellularized scaffolds had more intense and uniform Coomassie staining (Fig. 1a, b) than traditionally seeded scaffolds (Fig. 1e, f). In traditionally seeded scaffolds, fibers and stained clumps indicated an incomplete coverage of the construct by cells and ECM. Perhaps, traditionally seeded Schwann cells were not able to form stable contacts with fibers as well as the colony of cells in the 
Fig. 5 PC12 cell culture and neurite extension on a aligned PCL fibers with a single decellularized cell sheet, $\mathbf{b}$ random PCL fibers with a single decellularized cell sheet, and c aligned PCL fibers without decellularized sheet. Scale bars indicate distance of $200 \mu \mathrm{m}$. d Graph shows average measurement of neurite extensions for each condition. $(* p<0.0001)$
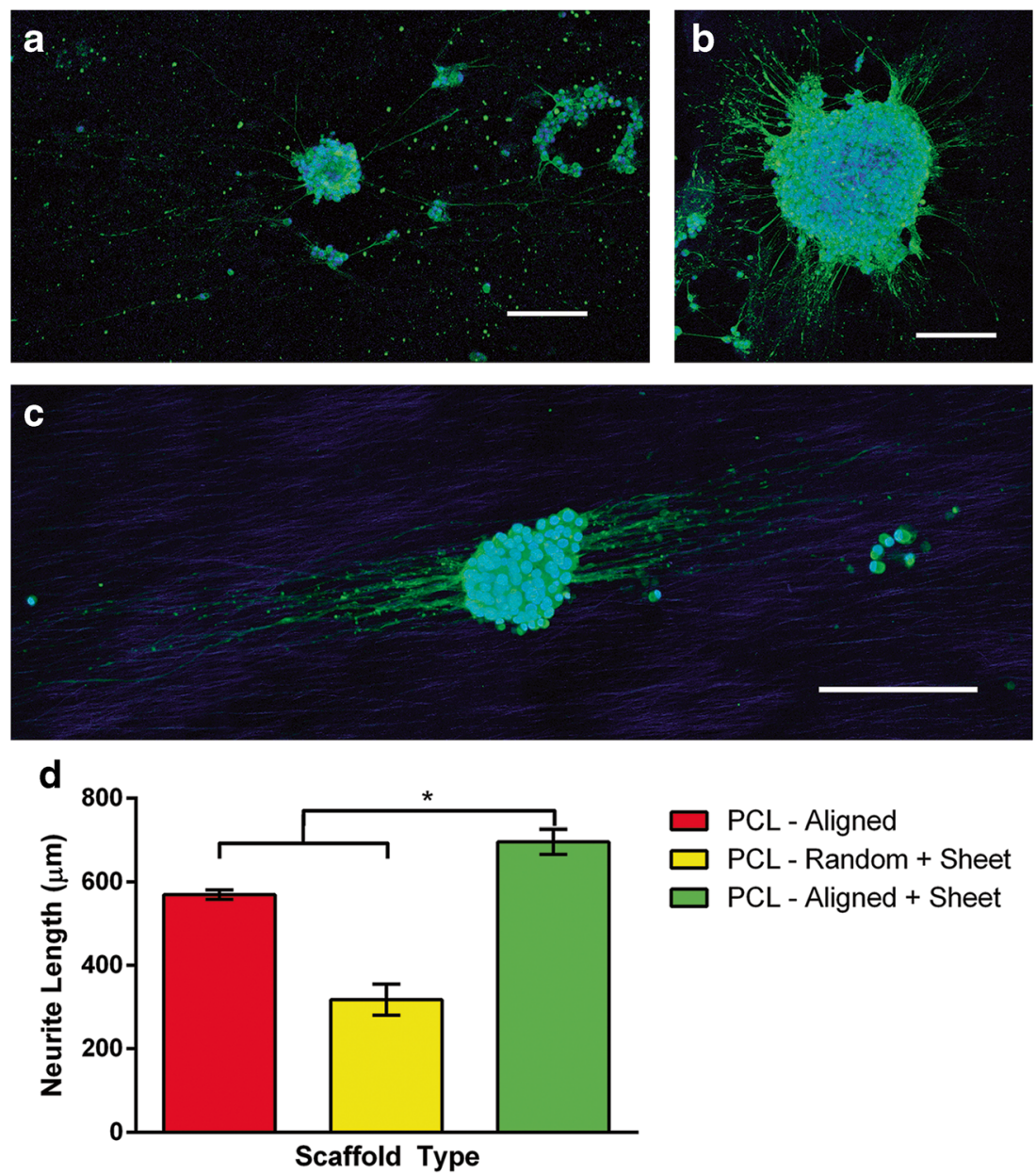

sheet. This could lead to detachment of cells and ECM throughout the decellularization process. The detergent treatment of cell sheets (Fig. 1c, d) was less effective in reducing nucleic acid content than in traditionally seeded scaffold (Fig. 1e, f). Nonetheless, the MB binding could also be accounted for presence of sulfated molecules that could bind to MB [36]. Staining for sulfated glycosaminoglycans could accurately test this assumption.

Ability to stack cell sheets illustrates one of advantages of using cell sheets to generate larger constructs. In Fig. 2a, three stacked cell sheets provided significantly more protein than a single sheet or traditionally seeded scaffolds. The amount of protein isolated from a single sheet directly correlated to the amount of protein isolated in three stacked sheets. In this respect, the detergent protocol selected did not result in loss of biological material in larger scaffolds. Conflict between Coomassie staining and BSA assay could be attributed to incomplete de-staining of scaffolds from the dye. Stacking of cell sheets hindered the removal of nucleic acids as the scaffold became larger. In Fig. 2b, traditionally seeded scaffolds had significantly less nucleic acids as opposed to scaffolds with one or more decellularized sheets. Surprisingly, stacking of cell sheets did not significantly increase the nucleic acid content in scaffolds. Use of other DNAquantifying assay kits could further confirm observation.

Neuronal constructs should provide a favorable microenvironment for glial as well as neuronal cells. In proliferation experiments, cells grown on scaffolds with decellularized Schwann cell sheets proliferated significantly better than on scaffolds without any biological molecules (Fig. 3). Although laminin has been used in numerous experiments to enhance cell attachment and proliferation [31,37-39], cells grown on scaffolds with covalently bound laminin through EDC/NHS mechanism did not show higher proliferation rates than cells grown on decellularized cell sheets. The experiment indicates the need for protein coating to enhance cell attachment and proliferation. Cells grown on decellularized Schwann cell sheets proliferated better perhaps due to the myriad of biological molecules, protein, glycoprotein, and carbohydrates, which the sheet provided as opposed to scaffolds with laminin alone. Likewise, EDC/NHS system is prone to denaturing native confirmation of laminin as opposed to mild detergent treatment. Decellularized cell sheets could provide a larger variety of biological material to the scaffold in more native condition than chemically bound protein. 
By using a semi-quantitative PCR method, the difference in proliferation rates of Schwann cells between scaffolds with decellularized cell sheets and scaffolds with nanofibers alone could be accounted for by elevated expression of Erbb2 gene, Fig. 4b. Pharmacological inhibition of Erbb signaling has been shown to reduce migration, proliferation, and the terminal differentiation of myelinating Schwann cells [40]. However, Schwann cells have been found to use a different mechanism and do not require neuregulin signaling through Erbb2 for proliferation and survival after nerve injury [41]. Further study at multiple time points might provide a more encompassing profile for this mechanism.

Another key difference revealed through PCR experiments was the slightly elevated laminin $\beta 1$ and $\gamma 1$ chain expression (Fig. 4c, e). The study of laminin expression is incomplete since there are eight other chain isoforms that would need to be tested [42]. This study focused on the expression pattern of only three laminin chains: $\alpha 2, \beta 1$, and $\gamma 1$. Laminin-211 is a main laminin isoform in skeletal muscle and neuromuscular junctions and basement membranes produced by Schwann cells [43, 44]. The $\gamma 1$ chain has also been linked to the ability of Schwann cells to differentiate and synthesize myelin proteins leading to sorting and myelination of axons [45]. Increased expression of the specific chains may suggest increased tendency to produce basement membrane components and form synapses. Elevation of $\beta 1$ and $\gamma 1$ laminin chains might also have significance in upregulation of $\beta 1$ integrin (Fig. 4f). Elevation of $\beta 1$ integrin coincides with increased expression of the laminin chains. By increasing expression of $\beta 1$ integrin, the protein deposited by decellularized Schwann cell sheets might have a significant impact on myelination [7,8]. This finding is complemented by increased expression of $\mathrm{P} 0$ in decellularized cell sheets (Fig. 4d). P0 is a major protein component of myelin sheath in peripheral nervous system, and upon myelination, $\mathrm{P} 0$ expression is upregulated [46]. Presence of basal lamina has been found to induce P0 gene expression in Schwann cells contacting axons destined for myelination [47]. This suggests that decellularized cell sheets may mimic the presence of basal lamina that contains molecular cues to induce differentiation and myelination of axons by Schwann cells.

Decellularized Schwann cell sheets along with directional physical guidance cues from aligned fibers optimally enhanced neurite extensions of differentiated PC12 cells. PC12 cells grown on decellularized sheets attached to random PCL fibers (Fig. 5b) had a higher neurite density when compared to cultures on scaffolds with decellularized sheets and aligned fibers (Fig. 5a). However, the latter culture had significantly longer neurite extension. Comparison to cultures grown on aligned PCL fibers alone (Fig. 5c) showed that physical cues dominate guidance of axons during regeneration. Likewise, the comparison between random and aligned physical cues provided by fibers (Fig. 5b, c) shows that fiber alignment plays a more significant role in neurite extension. Other groups had observed similar results [48-50]. Thickness of the matrix might have contributed to some of the loss of directionality of the axonal extensions visible in Fig. 5a. Further studies should focus on incorporation on molecular cues from Schwann cell ECM while preserving directional cues from nanofibrous structures.

\section{Conclusion}

ECM surrounding Schwann cells and neurons provides critical determinants of cellular phenotype during development as well as essential cues in stimulating and guiding regrowth. Combination of cell sheet technology and decellularization protocols allowed for development of a novel method to deposit myriad of proteins found in ECM simultaneously. Decellularization of these constructs proved to preserve majority of the ECM while retaining only few traces of nucleic acids. Layering of multiple cell sheets provided more extracellular milieu than traditional seeding method after decellularization. Additionally, Schwann cells seeded on the isolated matrix proliferated faster than on PCL or PCL/chitosan fibers. Covalently bound laminin enhanced proliferation rate but was not able to accommodate for the stimuli-rich microenviroment provided by the decellularized sheets. The proliferation and differentiation of Schwann cells grown on decellularized sheets were complemented by upregulation of Erbb2 and P0. Laminin expression of $\beta 1$ and $\gamma 1$ chains was also elevated. PC12 cells grown on decellularized sheets produced longer neurite extensions than aligned polycaprolactone fibers alone. These results encourage further studies into changes in genetic expression of Schwann cells at multiple time points as well as any increased secretion of neurotrophic factors. This novel method of ECM deposition warrants its use in future peripheral nerve regenerative studies.

\section{References}

1. Chernousov MA, Carey DJ. Schwann cell extracellular matrix molecules and their receptors. Histol Histopathol. 2000;15(2):593-601.

2. Frantz C, Stewart KM, Weaver VM. The extracellular matrix at a glance. J Cell Sci. 2010;123:4195-200.

3. Bunge MB, Williams AK, Wood PM, Uitto J, Jeffrey JJ. Comparison of nerve cell and nerve cell plus Schwann cell cultures, with particular emphasis on basal lamina and collagen formation. J Cell Biol. 1980;84(1):184-202.

4. Previtali SC, Nodari A, Taveggia C, Pardini C, Dina G, Villa A, et al. Expression of laminin receptors in Schwann cell differentiation: evidence for distinct roles. J Neurosci. 2003;23(13):5520-30.

5. Badylak SF. The extracellular matrix as a scaffold for tissue reconstruction. Semin Cell Dev Biol. 2002;13(5):377-83. 
6. Bissell MJ, Aggeler J. Dynamic reciprocity: how do extracellular matrix and hormones direct gene expression? Prog Clin Biol Res. 1987;249:251-62.

7. Di Summa PG, Kalbermatten DF, Raffoul W, Terenghi G, Kingham PJ. Extracellular matrix molecules enhance the neurotrophic effect of Schwann cell-like differentiated adipose-derived stem cells and increase cell survival under stress conditions. Tissue Eng A. 2013;19(3-4):368-79.

8. Dubový P. Schwann cells and endoneurial extracellular matrix molecules as potential cues for sorting of regenerated axons: a review. Anat Sci Int. 2004;79(4):198-208.

9. Evans GR, Brandt K, Katz S, Chauvin P, Otto L, Bogle M, et al. Bioactive poly(L-lactic acid) conduits seeded with Schwann cells for peripheral nerve regeneration. Biomaterials. 2002;23(3):841-8.

10. Fansa H, Dodic T, Wolf G, Schneider W, Keilhoff G. Tissue engineering of peripheral nerves: epineurial grafts with application of cultured Schwann cells. Microsurgery. 2003;23(1):72-7.

11. Keilhoff G, Fansa H, Schneider W, Wolf G. In vivo predegeneration of peripheral nerves: an effective technique to obtain activated Schwann cells for nerve conduits. J Neurosci Methods. 1999;89(1):17-24.

12. Karabekmez FE, Duymaz A, Moran SL. Early clinical outcomes with the use of decellularized nerve allograft for repair of sensory defects within the hand. Hand. 2009;4(3):245-9.

13. Hudson TW, Evans GR, Schmidt CE. Engineering strategies for peripheral nerve repair. Clin Plast Surg. 1999;26(4):61728.

14. Bellamkonda RV. Peripheral nerve regeneration: an opinion on channels, scaffolds and anisotropy. Biomaterials. 2006;27(19): 3515-8.

15. Martini R. Expression and functional roles of neural cell surface molecules and extracellular matrix components during development and regeneration of peripheral nerves. J Neurocytol. 1994;23(1):1-28.

16. Ide C, Osawa T, Tohyama K. Nerve regeneration through allogeneic nerve grafts, with special reference to the role of the Schwann cell basal lamina. Prog Neurobiol. 1990;34(1):1-38.

17. Dumont CE, Hentz VR. Enhancement of axon growth by detergentextracted nerve grafts. Transplantation. 1997;63(9):1210-5.

18. Sondell M, Lundborg G, Kanje M. Regeneration of the rat sciatic nerve into allografts made acellular through chemical extraction. Brain Res. 1998;795(1-2):44-54.

19. Monk KR, Wu J, Williams JP, Finney BA, Fitzgerald ME, Filippi MD, et al. Mast cells can contribute to axon-glial dissociation and fibrosis in peripheral nerve. Neuron Glia Biol. 2007;3(3):233-44.

20. Okano T, Yamada N, Sakai H, Sakurai Y. A novel recovery system for cultured cells using plasma-treated polystyrene dishes grafted with poly(N-isopropylacrylamide). J Biomed Mater Res. 1993;27(10):1243-51.

21. Hadlock TA, Sundback CA, Hunter DA, Vacanti JP, Cheney ML. A new artificial nerve graft containing rolled Schwann cell monolayers. Microsurgery. 2001;21(3):96-101.

22. Navarro X, Rodríguez FJ, Ceballos D, Verdú E. Engineering an artificial nerve graft for the repair of severe nerve injuries. Med Biol Eng Comput. 2003;41(2):220-6.

23. Okano T, Yamada N, Okuhara M, Sakai H, Sakurai Y. Mechanism of cell detachment from temperature-modulated, hydrophilic-hydrophobic polymer surfaces. Biomaterials. 1995;16(4):297-303.

24. Yamato M, Utsumi M, Kushida A, Konno C, Kikuchi A, Okano T. Thermo-responsive culture dishes allow the intact harvest of multilayered keratinocyte sheets without dispase by reducing temperature. Tissue Eng. 2001;7(4):473-80.

25. Nishida K, Yamato M, Hayashida Y, Watanabe K, Maeda N, Watanabe $\mathrm{H}$, et al. Functional bioengineered corneal epithelial sheet grafts from corneal stem cells expanded ex vivo on a temperature-responsive cell culture surface. Transplantation. 2004;77(3):379-85.

26. Nishida K, Yamato M, Hayashida Y, Watanabe K, Yamamoto K, Adachi E, et al. Corneal reconstruction with tissue-engineered cell sheets composed of autologous oral mucosal epithelium. N Engl J Med. 2004;351(12):1187-96.

27. Kushida A, Yamato M, Isoi Y, Kikuchi A, Okano T. A noninvasive transfer system for polarized renal tubule epithelial cell sheets using temperature-responsive culture dishes. Eur Cell Mater. 2005;10:23-40.

28. Kushida A, Yamato M, Konno C, Kikuchi A, Sakurai Y, Okano T. Temperature-responsive culture dishes allow nonenzymatic harvest of differentiated madin-darby canine kidney (MDCK) cell sheets. J Biomed Mater Res. 2000;51(2): 216-23.

29. Pesirikan N, Chang W, Zhang X, Xu J, Yu X. Characterization of Schwann cells in self-assembled sheets from thermoresponsive substrates. Tissue Eng A. 2013;19(13-14):1601-9.

30. Hudson TW, Liu SY, Schmidt CE. Engineering an improved acellular nerve graft via optimized chemical processing. Tissue Eng. 2004;10(9-10):1346-58.

31. Prabhakaran MP, Venugopal JR, Chyan TT, Hai LB, Chan $\mathrm{CK}$, Lim AY, et al. Electrospun biocomposite nanofibrous scaffolds for neural tissue engineering. Tissue Eng A. 2008;14(11):1787-97.

32. Wacker MJ, Godard MP. Analysis of one-step and two-step realtime RT-PCR using SuperScript III. J Biomol Tech. 2005;16(3): 266-71.

33. Marone M, Mozzetti S, De Ritis D, Pierelli L, Scambia G. Semiquantitative RT-PCR analysis to assess the expression levels of multiple transcripts from the same sample. Biol Proced Online. 2002;3:19-25.

34. Nagata S, Hanayama R, Kawane K. Autoimmunity and the clearance of dead cells. Cell. 2010;140(5):619-30.

35. Lo Leggio L, Williams RM, Jones R. Some effects of zona pellucida glycoproteins and sulfated polymers on the autoactivation of boar sperm proacrosin and activity of beta-acrosin. J Reprod Fertil. 1994;100(1):177-85.

36. Jiao QC, Liu Q, Sun $\mathrm{C}, \mathrm{He} H$. Investigation on the binding site in heparin by spectrophotometry. Talanta. 1999;48(5): 1095-101.

37. Dhoot NO, Tobias CA, Fischer I, Wheatley MA. Peptide-modified alginate surfaces as a growth permissive substrate for neurite outgrowth. J Biomed Mater Res A. 2004;71(2):191-200.

38. Bailey SB, Eichler ME, Villadiego A, Rich KM. The influence of fibronectin and laminin during Schwann cell migration and peripheral nerve regeneration through silicon chambers. J Neurocytol. 1993;22(3):176-84

39. Junka R, Valmikinathan CM, Kalyon DM, Yu X. Laminin functionalized biomimetic nanofibers for nerve tissue engineering. $\mathrm{J}$ Biomater Tissue Eng. 2013;3(4):494-502.

40. Lyons DA, Pogoda HM, Voas MG, Woods IG, Diamond B, Nix R, et al. Erbb3 and Erbb2 are essential for Schwann cell migration and myelination in zebrafish. Curr Biol. 2005;15(6):513-24.

41. Atanasoski S, Scherer SS, Sirkowski E, Leone D, Garratt AN, Birchmeier C, et al. ErbB2 signaling in Schwann cells is mostly dispensable for maintenance of myelinated peripheral nerves and proliferation of adult Schwann cells after injury. J Neurosci. 2006;26(7):2124-31.

42. Macdonald PR, Lustig A, Steinmetz MO, Kammerer RA. Laminin chain assembly is regulated by specific coiled-coil interactions. J Struct Biol. 2010;170(2):398-405.

43. Patton BL, Miner JH, Chiu AY, Sanes JR. Distribution and function of laminins in the neuromuscular system of developing, adult, and mutant mice. J Cell Biol. 1997;139(6):1507-21. 
44. Gawlik KI, Durbeej M. Skeletal muscle laminin and MDC1A: pathogenesis and treatment strategies. Skelet Muscle. 2011;1(1):9.

45. Chen Z, Strickland S. Laminin $\gamma 1$ is critical for Schwann cell differentiation, axon myelination, and regeneration in the peripheral nerve. J Cell Biol. 2003;163(4):889-99.

46. Peirano RI, Goerich DE, Riethmacher D, Wegner M. Protein zero gene expression is regulated by the glial transcription factor Sox 10 . Mol Cell Biol. 2000;20(9):3198-209.

47. Fernandez-Valle C, Fregien N, Wood PM, Bunge MB. Expression of the protein zero myelin gene in axon-related Schwann cells is linked to basal lamina formation. Development. 1993;119(3):867-80.
48. Neal RA, McClugage SG, Link MC, Sefcik LS, Ogle RC, Botchwey EA. Laminin nanofiber meshes that mimic morphological properties and bioactivity of basement membranes. J Tissue Eng C Methods. 2009;15(1):11-21.

49. Koh HS, Yong T, Chan CK, Ramakrishna S. Enhancement of neurite outgrowth using nano-structured scaffolds coupled with laminin. Biomaterials. 2008;29(26):3574-82.

50. Xie J, MacEwan MR, Li X, Sakiyama-Elbert SE, Xia Y. Neurite outgrowth on nanofiber scaffolds with different orders, structures, and surface properties. ACS Nano. 2009;3(5):1151-9. 